Supplementary Information

\title{
Proximity Effects of Substituents on Halogen Bond Strength
}

\author{
Jordan Lapp and Steve Scheiner* \\ Department of Chemistry and Biochemistry \\ Utah State University \\ Logan, Utah 84322-0300
}

\section{Table of Contents:}

$\begin{array}{ll}\text { 1. Dimers } & \text { S2 }\end{array}$

a. Cartesian coordinates for unsubstituted dimers $\quad$ S2

b. Cartesian coordinates for fluorinated dimers S6

$\begin{array}{ll}\text { c. Cartesian coordinates for cyanated dimers } & \text { S13 }\end{array}$

$\begin{array}{lr}\text { 2. Monomers } & \text { S22 }\end{array}$

$\begin{array}{ll}\text { a. Cartesian coordinates for unsubstituted monomers } & \text { S22 }\end{array}$

$\begin{array}{ll}\text { b. Cartesian coordinates for fluorinated monomers } & \text { S25 }\end{array}$

$\begin{array}{ll}\text { c. Cartesian coordinates for cyanated monomers } & \text { S30 }\end{array}$

$\begin{array}{ll}\text { d. Cartesian coordinates for ammonia } & \text { S37 }\end{array}$

\section{Coordinates Format:}

Atomic Number X-Coordinate $(\AA) \quad$ Y-Coordinate $(\AA) \quad$ Z-Coordinate $(\AA)$ 


\section{Dimer s}

\section{A. Unsubstituted Dimers}

\begin{tabular}{lrrr}
\multicolumn{4}{c}{ Alkene 1-H - - NH } \\
6 & -2.863440000 & 0.645792000 & 0.004029000 \\
6 & -1.998582000 & -0.363073000 & 0.009694000 \\
1 & -2.542297000 & 1.686187000 & -0.008388000 \\
1 & -2.308717000 & -1.406644000 & 0.022137000 \\
53 & 0.089329000 & -0.103193000 & -0.003746000 \\
1 & -3.935071000 & 0.442485000 & 0.012059000 \\
7 & 3.210051000 & 0.291292000 & 0.006854000 \\
1 & 3.693016000 & -0.468772000 & -0.464472000 \\
1 & 3.567362000 & 0.327093000 & 0.957675000 \\
1 & 3.493040000 & 1.153507000 & -0.450790000
\end{tabular}

Alkene 2-H - - - $\mathbf{N H}_{3}$

$\begin{array}{lrrr}6 & -2.163656000 & 0.228429000 & -0.001037000 \\ 6 & -1.165576000 & -0.661984000 & 0.006616000 \\ 1 & -1.948095000 & 1.299350000 & -0.014803000 \\ 1 & -1.350539000 & -1.735740000 & 0.020451000 \\ 53 & 0.864011000 & -0.141858000 & -0.005136000 \\ 7 & 3.910935000 & 0.620995000 & 0.012562000 \\ 1 & 4.481938000 & -0.078189000 & -0.454312000 \\ 1 & 4.258092000 & 0.702315000 & 0.964352000 \\ 1 & 4.091858000 & 1.509194000 & -0.447222000 \\ 6 & -3.569711000 & -0.171617000 & 0.008795000\end{array}$


Supplementary Information

$\begin{array}{lrrr}1 & -3.775750000 & -1.244598000 & 0.022698000 \\ 6 & -4.584904000 & 0.701145000 & 0.001627000 \\ 1 & -5.620685000 & 0.367269000 & 0.009241000 \\ 1 & -4.402852000 & 1.776070000 & -0.012148000\end{array}$

Alkene 3-H - - - $\mathrm{NH}_{3}$

$\begin{array}{llll}6 & 1.309451000 & 0.308399000 & -0.041565000\end{array}$

$\begin{array}{lllll}6 & 0.333809000 & -0.598803000 & -0.175339000\end{array}$

$1 \quad 1.063794000 \quad 1.355372000 \quad 0.150121000$

$1 \quad 0.545762000 \quad-1.650514000 \quad-0.365050000$

$\begin{array}{llll}53 & -1.706543000 & -0.144921000 & -0.033119000\end{array}$

$\begin{array}{llll}7 & -4.760054000 & 0.526492000 & 0.219054000\end{array}$

$\begin{array}{llll}1 & -5.301046000 & -0.288600000 & 0.495043000\end{array}$

$\begin{array}{llll}1 & -5.153683000 & 0.862483000 & -0.655824000\end{array}$

$\begin{array}{llll}1 & -4.928898000 & 1.245930000 & 0.916954000\end{array}$

$\begin{array}{llll}6 & 2.720338000 & -0.042191000 & -0.135960000\end{array}$

$1 \quad 2.961074000 \quad-1.090003000 \quad-0.333788000$

$\begin{array}{llll}6 & 3.716061000 & 0.854942000 & -0.013946000\end{array}$

$\begin{array}{llll}6 & 5.145288000 & 0.528919000 & -0.076001000\end{array}$

$\begin{array}{llll}1 & 5.802298000 & 1.306616000 & -0.472484000\end{array}$

$\begin{array}{llll}6 & 5.688444000 & -0.624585000 & 0.332544000\end{array}$

$\begin{array}{llll}1 & 5.083854000 & -1.407067000 & 0.791671000\end{array}$

$\begin{array}{llll}1 & 6.757079000 & -0.807269000 & 0.237073000\end{array}$

$\begin{array}{llll}1 & 3.456551000 & 1.908359000 & 0.119827000\end{array}$

Alkene 4-H - - - $\mathrm{NH}_{3}$

$\begin{array}{lllll}6 & 0.397582000 & -0.399136000 & 0.025466000\end{array}$

$\begin{array}{llll}6 & -0.541771000 & 0.534203000 & -0.175874000\end{array}$ 
Supplementary Information

\begin{tabular}{llll}
1 & 0.108816000 & -1.426027000 & 0.260867000 \\
1 & -0.289016000 & 1.567943000 & -0.409933000 \\
53 & -2.599724000 & 0.157331000 & -0.059860000 \\
7 & -5.676324000 & -0.377461000 & 0.181484000 \\
1 & -6.223734000 & 0.234839000 & -0.417266000 \\
1 & -5.986087000 & -0.214847000 & 1.135829000 \\
1 & -5.932134000 & -1.332169000 & -0.055700000 \\
6 & 1.821337000 & -0.106549000 & -0.050827000 \\
1 & 2.105727000 & 0.919601000 & -0.297864000 \\
6 & 2.777911000 & -1.035290000 & 0.147108000 \\
6 & 4.216908000 & -0.782148000 & 0.114598000 \\
1 & 4.855885000 & -1.629742000 & -0.144306000 \\
6 & 4.804036000 & 0.389993000 & 0.424711000 \\
1 & 2.466538000 & -2.067266000 & 0.328132000 \\
6 & 6.245819000 & 0.644996000 & 0.332737000 \\
1 & 6.656996000 & 1.360862000 & 1.048258000 \\
6 & 7.068510000 & 0.083722000 & -0.562281000 \\
1 & 8.135565000 & 0.297895000 & -0.557614000 \\
1 & 6.693439000 & -0.590010000 & -1.332924000 \\
\hline 6.185627000 & 1.203837000 & 0.810911000
\end{tabular}

Alkyne 1-H - - - $\mathrm{NH}_{3}$

$\begin{array}{llll}6 & 0.286343000 & -3.153054000 & 0.000000000\end{array}$

$\begin{array}{llll}6 & 0.181601000 & -1.946308000 & 0.000000000\end{array}$

$\begin{array}{llll}1 & 0.377905000 & -4.219368000 & 0.000000000\end{array}$

$\begin{array}{llll}53 & 0.000000000 & 0.068693000 & 0.000000000\end{array}$

$\begin{array}{llll}7 & -0.306201000 & 3.004735000 & 0.000000000\end{array}$

$\begin{array}{llll}1 & 0.590662000 & 3.483266000 & 0.000000000\end{array}$ 
Supplementary Information

$\begin{array}{lrrr}1 & -0.816413000 & 3.329187000 & 0.817250000 \\ 1 & -0.816413000 & 3.329187000 & -0.817250000\end{array}$

\begin{tabular}{lrrr}
\multicolumn{4}{c}{ Alkyne 2-H - - NH 3} \\
6 & -2.433086000 & -0.000213000 & 0.000085000 \\
6 & -1.215803000 & -0.002008000 & 0.000909000 \\
53 & 0.802132000 & -0.002012000 & 0.000377000 \\
7 & 3.736843000 & 0.006859000 & -0.001531000 \\
1 & 4.119985000 & -0.816515000 & 0.455053000 \\
1 & 4.110664000 & 0.817707000 & 0.484940000 \\
1 & 4.114778000 & 0.026249000 & -0.945092000 \\
6 & -3.812770000 & 0.001965000 & -0.000150000 \\
6 & -5.025324000 & 0.004299000 & -0.001098000 \\
1 & -6.094436000 & 0.006952000 & -0.002621000
\end{tabular}

\begin{tabular}{llll}
\multicolumn{4}{c}{ Alkyne 3-H - - NH } \\
6 & 0.003444000 & -0.003762000 & -1.573615000 \\
6 & 0.004133000 & -0.001906000 & -0.354643000 \\
53 & 0.004771000 & 0.000651000 & 1.661704000 \\
7 & -0.000302000 & 0.002286000 & 4.587750000 \\
1 & -0.044007000 & 0.944523000 & 4.967596000 \\
1 & 0.832187000 & -0.433732000 & 4.975457000 \\
1 & -0.801077000 & -0.503186000 & 4.957729000 \\
6 & -0.006716000 & 0.000141000 & -2.944563000 \\
6 & 0.026932000 & -0.019213000 & -4.163361000 \\
6 & 0.005161000 & -0.008769000 & -5.536993000 \\
6 & 0.004946000 & -0.008344000 & -6.750277000 \\
1 & -0.001636000 & -0.004120000 & -7.819996000
\end{tabular}


Supplementary Information

\begin{tabular}{lrrr}
\multicolumn{4}{c}{ Iodobenzene - - NH } \\
6 & 3.808932000 & -0.008750000 & 0.008502000 \\
6 & 3.112966000 & 1.199315000 & 0.005953000 \\
6 & 3.104493000 & -1.211947000 & 0.005630000 \\
1 & 3.656370000 & 2.143593000 & 0.008265000 \\
1 & 3.641167000 & -2.160087000 & 0.007686000 \\
6 & 1.717688000 & 1.209915000 & 0.000750000 \\
6 & 1.709267000 & -1.212577000 & 0.000433000 \\
1 & 1.175786000 & 2.154085000 & -0.000986000 \\
6 & 1.021414000 & 0.001093000 & -0.001948000 \\
1 & 4.897765000 & -0.012594000 & 0.012815000 \\
\hline 53 & -1.093148000 & 0.006568000 & -0.006815000 \\
7 & -4.231319000 & -0.015632000 & 0.018856000 \\
1 & -4.588881000 & 0.215291000 & 0.941778000 \\
1 & -4.593857000 & -0.934527000 & -0.220197000 \\
1 & -4.641430000 & 0.646105000 & -0.634551000 \\
1 & 1.160612000 & -2.152822000 & -0.001543000
\end{tabular}

\section{B. Fluorinated Dimers}

\begin{tabular}{cccc}
\multicolumn{4}{c}{ Alkene 1-F - - NH } \\
6 & 2.294825000 & 0.109945000 & 0.001655000 \\
6 & 1.305399000 & -0.766255000 & 0.002250000 \\
1 & 1.545526000 & -1.824189000 & 0.006109000 \\
53 & -0.707075000 & -0.205345000 & -0.002489000 \\
7 & -3.635887000 & 0.546707000 & 0.006383000 \\
1 & -4.087302000 & 0.284297000 & 0.878479000
\end{tabular}


Supplementary Information

$\begin{array}{lrrr}1 & -4.154952000 & 0.099704000 & -0.744649000 \\ 1 & -3.758340000 & 1.549928000 & -0.102521000 \\ 9 & 3.580758000 & -0.214585000 & 0.005784000 \\ 9 & 2.172565000 & 1.423970000 & -0.002852000\end{array}$

Alkene 2-F - - - $\mathrm{NH}_{3}$

$\begin{array}{llll}6 & -1.392607000 & -0.083284000 & -0.000901000\end{array}$

$\begin{array}{llll}6 & -0.323828000 & -0.886858000 & 0.000579000\end{array}$

$\begin{array}{llll}1 & -1.268892000 & 1.000457000 & -0.004284000\end{array}$

$\begin{array}{llll}1 & -0.414902000 & -1.972439000 & 0.004001000\end{array}$

$53 \quad 1.650761000 \quad-0.184486000 \quad-0.002073000$

$\begin{array}{llll}7 & 4.576182000 & 0.901836000 & 0.006626000\end{array}$

$\begin{array}{llll}1 & 5.245436000 & 0.236371000 & -0.370745000\end{array}$

$\begin{array}{llll}1 & 4.883516000 & 1.132071000 & 0.947685000\end{array}$

$\begin{array}{llll}1 & 4.654342000 & 1.749869000 & -0.548202000\end{array}$

$\begin{array}{llll}6 & -2.754710000 & -0.604835000 & 0.002310000\end{array}$

$\begin{array}{llll}1 & -2.935225000 & -1.677199000 & 0.006117000\end{array}$

$\begin{array}{llll}6 & -3.825288000 & 0.181774000 & 0.001139000\end{array}$

$\begin{array}{llll}9 & -3.801297000 & 1.504330000 & -0.003394000\end{array}$

$\begin{array}{llll}9 & -5.077513000 & -0.242662000 & 0.004523000\end{array}$

Alkene 3-F - - - $\mathrm{NH}_{3}$

$\begin{array}{lrrr}6 & 0.471842000 & -0.460975000 & -0.015800000 \\ 6 & -0.449934000 & 0.510913000 & -0.018481000 \\ 1 & 0.160908000 & -1.508321000 & -0.013460000 \\ 1 & -0.179194000 & 1.566296000 & -0.020629000 \\ 53 & -2.514131000 & 0.148866000 & -0.011124000 \\ 7 & -5.602081000 & -0.375930000 & 0.053430000\end{array}$


Supplementary Information

$\begin{array}{lrrr}1 & -6.117325000 & 0.372216000 & -0.402595000 \\ 1 & -5.935554000 & -0.425547000 & 1.012306000 \\ 1 & -5.872306000 & -1.243714000 & -0.401383000 \\ 6 & 1.901871000 & -0.186921000 & -0.013274000 \\ 1 & 2.205570000 & 0.860397000 & -0.014116000 \\ 6 & 2.819352000 & -1.175268000 & -0.008153000 \\ 6 & 4.273778000 & -1.051050000 & -0.000971000 \\ 1 & 4.875679000 & -1.956183000 & -0.003768000 \\ 6 & 4.974481000 & 0.079144000 & 0.011421000 \\ 1 & 2.455387000 & -2.204194000 & -0.008486000 \\ 9 & 6.295842000 & 0.141260000 & 0.019594000 \\ 9 & 4.473269000 & 1.301587000 & 0.018097000\end{array}$

Alkene 4-F - - - $\mathrm{NH}_{3}$

$\begin{array}{llll}6 & 0.517735000 & 0.344488000 & 0.105152000\end{array}$

$6 \quad 1.469143000 \quad-0.537773000 \quad-0.229310000$

$\begin{array}{llll}1 & 0.794238000 & 1.334863000 & 0.474114000\end{array}$

$\begin{array}{llll}1 & 1.228720000 & -1.534239000 & -0.599076000\end{array}$

$\begin{array}{llll}53 & 3.522118000 & -0.145135000 & -0.086976000\end{array}$

$\begin{array}{llll}7 & 6.587837000 & 0.413488000 & 0.195800000\end{array}$

$1 \quad 7.150227000 \quad-0.183653000 \quad-0.404421000$

$\begin{array}{llll}1 & 6.885197000 & 0.238417000 & 1.151913000\end{array}$

$\begin{array}{llll}1 & 6.837864000 & 1.374337000 & -0.022110000\end{array}$

$\begin{array}{llll}6 & -0.901558000 & 0.041393000 & -0.000272000\end{array}$

$1 \quad-1.169932000 \quad-0.946239000 \quad-0.384572000$

$\begin{array}{llll}6 & -1.872234000 & 0.919039000 & 0.324507000\end{array}$

$\begin{array}{llll}6 & -3.308635000 & 0.656294000 & 0.258785000\end{array}$

$\begin{array}{llll}1 & -3.951424000 & 1.524540000 & 0.113822000\end{array}$ 
Supplementary Information

$\begin{array}{lrrr}6 & -3.858138000 & -0.567615000 & 0.393635000 \\ 1 & -1.575758000 & 1.922832000 & 0.638591000 \\ 6 & -5.270752000 & -0.925762000 & 0.300965000 \\ 1 & -5.568800000 & -1.941117000 & 0.550122000 \\ 6 & -6.269012000 & -0.132897000 & -0.078326000 \\ 1 & -3.196799000 & -1.406210000 & 0.617865000 \\ 9 & -6.160502000 & 1.134004000 & -0.437666000 \\ 9 & -7.535045000 & -0.508314000 & -0.156548000\end{array}$

Alkyne 1-F - - - $\mathrm{NH}_{3}$

$\begin{array}{llll}6 & -2.734978000 & -0.001599000 & -0.000487000\end{array}$

$\begin{array}{llll}6 & -1.529486000 & 0.001011000 & 0.000598000\end{array}$

$\begin{array}{llll}53 & 0.497656000 & 0.002042000 & 0.000450000\end{array}$

$\begin{array}{llll}7 & 3.426342000 & -0.006114000 & -0.001664000\end{array}$

$\begin{array}{llll}1 & 3.806996000 & 0.455955000 & -0.823320000\end{array}$

$\begin{array}{llll}1 & 3.799585000 & -0.951657000 & 0.008871000\end{array}$

$\begin{array}{llll}1 & 3.804297000 & 0.472859000 & 0.811380000\end{array}$

$9 \quad-4.020472000 \quad-0.004339000 \quad-0.001090000$

Alkyne 2-F - - - $\mathrm{NH}_{3}$

$\begin{array}{lrrr}6 & -1.906536000 & -0.000607000 & -0.000393000 \\ 6 & -0.690021000 & -0.001373000 & -0.000422000 \\ 53 & 1.328766000 & -0.001452000 & -0.000268000 \\ 7 & 4.265616000 & 0.005081000 & 0.001131000 \\ 1 & 4.650374000 & -0.933676000 & -0.064973000 \\ 1 & 4.640420000 & 0.420761000 & 0.849809000 \\ 1 & 4.640545000 & 0.535909000 & -0.780566000 \\ 6 & -3.286857000 & 0.000374000 & -0.000126000\end{array}$


Supplementary Information

$\begin{array}{llll}6 & -4.492843000 & 0.001273000 & 0.000219000 \\ 9 & -5.773077000 & 0.002269000 & 0.000708000\end{array}$

\begin{tabular}{llll}
\multicolumn{4}{c}{ Alkyne 3-F - - NH 3} \\
6 & 0.005404000 & 0.004290000 & -1.570270000 \\
6 & 0.006482000 & 0.000480000 & -0.351267000 \\
53 & 0.004099000 & -0.001650000 & 1.665620000 \\
7 & -0.004203000 & 0.000328000 & 4.593419000 \\
1 & 0.000717000 & 0.945683000 & 4.967619000 \\
1 & 0.811392000 & -0.473413000 & 4.972944000 \\
1 & -0.822739000 & -0.466148000 & 4.975634000 \\
6 & 0.000871000 & 0.014199000 & -2.942219000 \\
6 & 0.012912000 & -0.028006000 & -4.163029000 \\
6 & 0.005699000 & -0.009800000 & -5.536312000 \\
6 & 0.003890000 & -0.015008000 & -6.728887000 \\
9 & -0.000722000 & -0.008863000 & -8.006788000
\end{tabular}

\begin{tabular}{lrrr}
\multicolumn{4}{c}{ Ortho-Iodobenzene-F - - $\mathbf{N H}_{3}$} \\
6 & -3.721803000 & -0.313815000 & 0.008262000 \\
6 & -2.979305000 & -1.494448000 & 0.005070000 \\
6 & -3.072382000 & 0.918166000 & 0.006014000 \\
1 & -3.483709000 & -2.459214000 & 0.006950000 \\
1 & -3.618680000 & 1.859509000 & 0.008535000 \\
6 & -1.586139000 & -1.444982000 & -0.000085000 \\
6 & -1.683967000 & 0.945210000 & 0.000741000 \\
1 & -1.004560000 & -2.365346000 & -0.002145000 \\
6 & -0.920392000 & -0.217772000 & -0.002285000 \\
1 & -4.809798000 & -0.348903000 & 0.012665000
\end{tabular}


Supplementary Information

$\begin{array}{rrrr}53 & 1.182728000 & -0.120056000 & -0.006221000 \\ 9 & -1.070702000 & 2.142903000 & -0.000946000 \\ 7 & 4.252587000 & -0.004857000 & 0.017920000 \\ 1 & 4.626778000 & -0.241832000 & 0.932747000 \\ 1 & 4.564475000 & 0.938339000 & -0.196923000 \\ 1 & 4.693039000 & -0.625874000 & -0.655325000\end{array}$

Meta-Iodobenzene-F - - - $\mathrm{NH}_{3}$

$\begin{array}{llll}6 & 3.503673000 & 0.571567000 & 0.009244000 \\ 6 & 2.666132000 & 1.685821000 & 0.008237000 \\ 6 & 2.913260000 & -0.683327000 & 0.003665000 \\ 1 & 3.100347000 & 2.684778000 & 0.012748000 \\ 6 & 1.278698000 & 1.539987000 & 0.002052000 \\ 6 & 1.538075000 & -0.871439000 & -0.002535000 \\ 1 & 0.635334000 & 2.416950000 & 0.001680000 \\ 6 & 0.722594000 & 0.260120000 & -0.003216000 \\ 53 & -1.376204000 & 0.019393000 & -0.008403000 \\ 7 & -4.449920000 & -0.379214000 & 0.024305000 \\ 1 & -4.796999000 & -0.416993000 & 0.978890000 \\ 1 & -4.715119000 & -1.252128000 & -0.423738000 \\ 1 & -4.957282000 & 0.364109000 & -0.448127000 \\ 1 & 4.587598000 & 0.660330000 & 0.014337000 \\ 1 & 1.130813000 & -1.879884000 & -0.006440000 \\ 9 & 3.707664000 & -1.774095000 & 0.004579000\end{array}$

Para-Iodobenzene-F - - - $\mathrm{NH}_{3}$

$\begin{array}{llll}6 & -3.407994000 & -0.004429000 & 0.004852000\end{array}$

$\begin{array}{llll}6 & -2.737463000 & -1.218114000 & 0.002598000\end{array}$ 
Supplementary Information

$\begin{array}{lrrr}6 & -2.743192000 & 1.212379000 & 0.002910000 \\ 1 & -3.302784000 & -2.147905000 & 0.004284000 \\ 1 & -3.312951000 & 2.139449000 & 0.004822000 \\ 6 & -1.342825000 & -1.209553000 & -0.001562000 \\ 6 & -1.348483000 & 1.210563000 & -0.001267000 \\ 1 & -0.797794000 & -2.151665000 & -0.003146000 \\ 6 & -0.650798000 & 0.002137000 & -0.003447000 \\ 9 & -4.757910000 & -0.007592000 & 0.009164000 \\ 53 & 1.462695000 & 0.005572000 & -0.006518000 \\ 1 & -0.808023000 & 2.155298000 & -0.002621000 \\ 7 & 4.577337000 & -0.015718000 & 0.020671000 \\ 1 & 4.936072000 & 0.215536000 & 0.943091000 \\ 1 & 4.984560000 & 0.647704000 & -0.632881000 \\ 1 & 4.942470000 & -0.933261000 & -0.219773000\end{array}$

Ortho-Bisub-Iodobenzene-F - - - $\mathrm{NH}_{3}$

$\begin{array}{llll}6 & 3.662825000 & -0.010847000 & 0.006907000\end{array}$

$\begin{array}{llll}6 & 2.977271000 & 1.201615000 & 0.004702000\end{array}$

$\begin{array}{llll}6 & 2.967600000 & -1.217784000 & 0.004614000\end{array}$

$\begin{array}{llll}1 & 3.493738000 & 2.158736000 & 0.006579000\end{array}$

$\begin{array}{llll}1 & 3.476205000 & -2.179126000 & 0.006410000\end{array}$

$\begin{array}{llll}6 & 1.589229000 & 1.180556000 & 0.000345000\end{array}$

$\begin{array}{llll}6 & 1.579810000 & -1.185422000 & 0.000249000\end{array}$

$\begin{array}{llll}6 & 0.849454000 & 0.000476000 & -0.001944000\end{array}$

$\begin{array}{llll}1 & 4.750969000 & -0.015207000 & 0.010575000\end{array}$

$\begin{array}{llll}53 & -1.251688000 & 0.006816000 & -0.004681000\end{array}$

$9 \quad 0.915355000 \quad-2.353516000 \quad-0.001586000$

$\begin{array}{llll}7 & -4.265464000 & -0.011750000 & 0.014401000\end{array}$ 
Supplementary Information

$\begin{array}{llll}1 & -4.631913000 & 0.222954000 & 0.932964000 \\ 1 & -4.627609000 & -0.930492000 & -0.226195000 \\ 1 & -4.665941000 & 0.649938000 & -0.645116000 \\ 9 & 0.934102000 & 2.353805000 & -0.001434000\end{array}$

\title{
Meta-Bisub-Iodobenzene-F - - - $\mathrm{NH}_{3}$
}

\begin{tabular}{lrrr}
6 & -3.315297000 & 0.005137000 & 0.006431000 \\
6 & -2.586678000 & -1.175527000 & 0.003571000 \\
6 & -2.581004000 & 1.182274000 & 0.003452000 \\
6 & -1.198803000 & -1.215180000 & -0.001688000 \\
6 & -1.192973000 & 1.215046000 & -0.001797000 \\
1 & -0.684677000 & -2.172982000 & -0.003385000 \\
6 & -0.510975000 & -0.001710000 & -0.004249000 \\
53 & 1.600737000 & -0.005620000 & -0.008012000 \\
7 & 4.672233000 & 0.011593000 & 0.024138000 \\
1 & 5.021901000 & 0.003035000 & 0.978503000 \\
1 & 5.044287000 & 0.845752000 & -0.421785000 \\
1 & 5.082600000 & -0.787884000 & -0.450771000 \\
\hline 1 & -4.401616000 & 0.007617000 & 0.010849000 \\
1 & -0.674143000 & 2.170286000 & -0.003580000 \\
9 & -3.250842000 & 2.350015000 & 0.006084000 \\
9 & -3.262343000 & -2.339941000 & 0.006307000
\end{tabular}

\section{C. Cyanated Dimers}

\author{
Alkene 1-CN - - - $\mathrm{NH}_{3}$ \\ $\begin{array}{llll}6 & -2.023192000 & 0.026205000 & -0.000100000\end{array}$ \\ $\begin{array}{llll}6 & -0.991680000 & -0.841802000 & -0.000355000\end{array}$
}


Supplementary Information

$\begin{array}{lrrr}1 & -1.204265000 & -1.910287000 & -0.000403000 \\ 53 & 1.015602000 & -0.304953000 & -0.000073000 \\ 7 & 3.883536000 & 0.463801000 & 0.000658000 \\ 1 & 4.523135000 & -0.305138000 & -0.181772000 \\ 1 & 4.156169000 & 0.871400000 & 0.891494000 \\ 1 & 4.057574000 & 1.169462000 & -0.710621000 \\ 6 & -1.845169000 & 1.454577000 & -0.000069000 \\ 6 & -3.378165000 & -0.466387000 & 0.000199000 \\ 7 & -1.700848000 & 2.601916000 & -0.000154000 \\ 7 & -4.458441000 & -0.879783000 & 0.000512000\end{array}$

Alkene 2-CN - - - $\mathrm{NH}_{3}$

$\begin{array}{lrrr}6 & -1.007450000 & -0.122066000 & -0.004737000 \\ 6 & 0.068348000 & -0.927677000 & -0.004861000 \\ 1 & -0.900086000 & 0.963858000 & -0.006754000 \\ 1 & -0.036623000 & -2.013284000 & -0.002819000 \\ 53 & 2.034353000 & -0.233453000 & -0.004822000 \\ 7 & 4.865688000 & 0.861213000 & 0.020823000 \\ 1 & 5.484758000 & 0.403660000 & -0.643175000 \\ 1 & 5.298559000 & 0.774165000 & 0.936744000 \\ 1 & 4.862835000 & 1.851073000 & -0.211569000 \\ 6 & -2.339558000 & -0.682074000 & -0.000326000 \\ 1 & -2.440425000 & -1.768377000 & 0.002778000 \\ 6 & -3.480791000 & 0.052805000 & 0.001391000 \\ 6 & -3.447441000 & 1.490521000 & -0.002808000 \\ 6 & -4.769426000 & -0.582439000 & 0.008128000 \\ 7 & -3.384629000 & 2.645959000 & -0.006561000 \\ 7 & -5.799893000 & -1.108955000 & 0.014255000\end{array}$


Supplementary Information

\begin{tabular}{lrrr}
\multicolumn{4}{c}{ Alkene 3-CN - - NH } \\
6 & -0.057724000 & -0.588079000 & 0.016424000 \\
6 & 0.839236000 & 0.412005000 & 0.022284000 \\
1 & 0.272367000 & -1.628651000 & 0.009802000 \\
1 & 0.527414000 & 1.456798000 & 0.028873000 \\
53 & 2.905551000 & 0.113224000 & 0.010626000 \\
7 & 5.943454000 & -0.318300000 & -0.056346000 \\
1 & 6.439220000 & 0.434722000 & 0.413552000 \\
1 & 6.286627000 & -0.346371000 & -1.012888000 \\
1 & 6.233070000 & -1.185606000 & 0.387866000 \\
6 & -1.481602000 & -0.322981000 & 0.015365000 \\
1 & -1.783248000 & 0.724544000 & 0.020248000 \\
6 & -2.401555000 & -1.320733000 & 0.006956000 \\
6 & -3.840817000 & -1.198573000 & 0.001012000 \\
\hline 1 & -4.387899000 & -2.142145000 & 0.000882000 \\
6 & -4.631989000 & -0.089778000 & -0.006427000 \\
\hline 6 & -2.034284000 & -2.347615000 & 0.003896000 \\
\hline 7 & -4.134491000 & 1.255337000 & -0.009490000 \\
\hline 6 & -6.061962000 & -0.236005000 & -0.013268000 \\
\hline 6 & -3.720237000 & 2.336625000 & -0.011912000 \\
\hline 6 & -0.366000000 & -0.019250000
\end{tabular}

Alkene 4-CN - - - $\mathrm{NH}_{3}$

$\begin{array}{llll}6 & 0.976566000 & 0.323162000 & 0.050844000\end{array}$

$6 \quad 1.940042000 \quad-0.584423000 \quad-0.170794000$

$\begin{array}{llll}1 & 1.234622000 & 1.355359000 & 0.295928000\end{array}$

$1 \quad 1.703288000 \quad-1.619818000 \quad-0.416347000$ 
Supplementary Information

\begin{tabular}{lrrr}
53 & 3.984574000 & -0.165454000 & -0.061503000 \\
7 & 6.997596000 & 0.419338000 & 0.186544000 \\
1 & 7.564309000 & -0.176125000 & -0.411574000 \\
1 & 7.305512000 & 0.257389000 & 1.141824000 \\
1 & 7.236222000 & 1.380869000 & -0.041781000 \\
6 & -0.430327000 & -0.022100000 & -0.015661000 \\
1 & -0.667396000 & -1.058705000 & -0.267575000 \\
6 & -1.423979000 & 0.869679000 & 0.199386000 \\
6 & -2.847178000 & 0.580769000 & 0.155227000 \\
1 & -3.497245000 & 1.453792000 & 0.127537000 \\
6 & -3.395889000 & -0.661737000 & 0.171987000 \\
1 & -1.151997000 & 1.906898000 & 0.400914000 \\
6 & -4.798350000 & -0.999050000 & 0.122427000 \\
\hline 1 & -5.021049000 & -2.064540000 & 0.191541000 \\
6 & -5.899522000 & -0.204052000 & 0.002096000 \\
\hline 1 & -2.734461000 & -1.524338000 & 0.251510000 \\
6 & -5.858727000 & 1.224823000 & -0.111630000 \\
\hline 7 & -7.206381000 & -0.802120000 & -0.017255000 \\
\hline 6 & -5.814864000 & 2.378626000 & -0.201093000 \\
\hline 6.252982000 & -1.296740000 & -0.032892000
\end{tabular}

Alkyne 1-CN - - - $\mathrm{NH}_{3}$

$\begin{array}{lrrr}6 & -2.443454000 & -0.000656000 & -0.000232000 \\ 6 & -1.228094000 & -0.000938000 & -0.000175000 \\ 53 & 0.792038000 & -0.000476000 & -0.000346000 \\ 7 & 3.678073000 & 0.001849000 & 0.001036000 \\ 1 & 4.055295000 & 0.032717000 & 0.944869000 \\ 1 & 4.057406000 & 0.803892000 & -0.495991000\end{array}$


Supplementary Information

$\begin{array}{lrrr}1 & 4.058503000 & -0.829784000 & -0.443218000 \\ 6 & -3.826744000 & 0.000602000 & 0.000298000 \\ 7 & -4.986571000 & 0.001634000 & 0.000873000\end{array}$

Alkyne 2-CN - - - $\mathrm{NH}_{3}$

$\begin{array}{lrrr}6 & -1.578779000 & -0.000310000 & -0.002641000 \\ 6 & -0.359519000 & -0.000985000 & -0.002708000 \\ 53 & 1.658043000 & -0.001794000 & -0.001258000 \\ 7 & 4.550344000 & 0.005774000 & 0.006346000 \\ 1 & 4.926276000 & -0.217626000 & 0.924431000 \\ 1 & 4.926826000 & 0.913465000 & -0.254893000 \\ 1 & 4.936355000 & -0.673241000 & -0.644597000 \\ 6 & -2.948847000 & 0.014039000 & -0.003881000 \\ 6 & -4.165513000 & -0.011067000 & 0.001745000 \\ 6 & -5.544606000 & -0.002431000 & 0.002772000 \\ 7 & -6.704935000 & 0.005229000 & 0.003655000\end{array}$

Alkyne 3-CN - - - $\mathrm{NH}_{3}$

$\begin{array}{lrrr}6 & -0.621328000 & -0.022992000 & 0.004190000 \\ 6 & 0.598697000 & -0.024468000 & 0.023753000 \\ 53 & 2.614949000 & -0.029563000 & 0.051401000 \\ 7 & 5.514150000 & -0.047499000 & 0.084754000 \\ 1 & 5.883691000 & -0.972153000 & 0.290756000 \\ 1 & 5.891096000 & 0.584801000 & 0.786236000 \\ 1 & 5.906945000 & 0.235567000 & -0.809506000 \\ 6 & -1.988339000 & -0.001714000 & -0.005152000 \\ 6 & -3.209023000 & -0.029913000 & 0.020116000 \\ 6 & -4.572856000 & -0.023935000 & -0.013759000\end{array}$


Supplementary Information

$\begin{array}{llll}6 & -5.789898000 & 0.010724000 & 0.003061000 \\ 6 & -7.168079000 & 0.020160000 & -0.011030000 \\ 7 & -8.328353000 & 0.030361000 & -0.024731000\end{array}$

\begin{tabular}{lrrr}
\multicolumn{4}{c}{ Ortho-Iodobenzene-CN - - NH } \\
6 & -3.707485000 & -0.540025000 & 0.002662000 \\
6 & -2.927131000 & -1.696658000 & 0.001192000 \\
6 & -3.090575000 & 0.704537000 & 0.002123000 \\
1 & -3.403274000 & -2.676470000 & 0.001575000 \\
6 & -1.536111000 & -1.611535000 & -0.000577000 \\
6 & -1.691164000 & 0.796497000 & 0.000268000 \\
1 & -0.933981000 & -2.517893000 & -0.001485000 \\
6 & -0.904979000 & -0.367226000 & -0.000977000 \\
\hline 53 & 1.201647000 & -0.270884000 & -0.001587000 \\
7 & 4.225431000 & -0.027198000 & 0.005511000 \\
\hline 1 & 4.631993000 & -0.143766000 & 0.929873000 \\
\hline 1 & 4.426222000 & 0.924979000 & -0.289103000 \\
\hline 1 & 4.729732000 & -0.646376000 & -0.623181000 \\
\hline 1 & -4.793553000 & -0.607854000 & 0.004250000 \\
\hline 1 & -3.679243000 & 1.619937000 & 0.003208000 \\
6 & -1.093965000 & 2.108147000 & -0.000284000 \\
\hline 7 & -0.647822000 & 3.176038000 & -0.000864000
\end{tabular}

Meta-Iodobenzene-CN - - - $\mathrm{NH}_{3}$

$\begin{array}{llll}6 & 3.219989000 & 1.072053000 & 0.000369000\end{array}$

$\begin{array}{llll}6 & 2.262151000 & 2.080783000 & 0.000300000\end{array}$

$\begin{array}{llll}6 & 2.801350000 & -0.262753000 & 0.000039000\end{array}$

$\begin{array}{llll}1 & 2.573886000 & 3.124087000 & 0.000548000\end{array}$ 
Supplementary Information

$\begin{array}{llll}6 & 0.902794000 & 1.766954000 & -0.000048000 \\ 6 & 1.439540000 & -0.591097000 & -0.000303000 \\ 1 & 0.160217000 & 2.563026000 & -0.000084000 \\ 6 & 0.494551000 & 0.431439000 & -0.000313000 \\ 53 & -1.564105000 & -0.037017000 & -0.000180000 \\ 7 & -4.555431000 & -0.702373000 & 0.001031000 \\ 1 & -4.855387000 & -1.091652000 & 0.890982000 \\ 1 & -4.779675000 & -1.389498000 & -0.713765000 \\ 1 & -5.136578000 & 0.112238000 & -0.177671000 \\ 1 & 4.282288000 & 1.305856000 & 0.000674000 \\ 1 & 1.134951000 & -1.635512000 & -0.000540000 \\ 6 & 3.782176000 & -1.320031000 & 0.000108000 \\ 7 & 4.570080000 & -2.167731000 & 0.000178000\end{array}$

Para-Iodobenzene-CN - - - $\mathrm{NH}_{3}$

$\begin{array}{lrrr}6 & 3.180566000 & 0.002674000 & 0.002582000 \\ 6 & 2.481549000 & 1.214937000 & -0.000045000 \\ 6 & 2.486516000 & -1.212455000 & 0.000367000 \\ 1 & 3.030342000 & 2.154732000 & 0.001653000 \\ 6 & 1.090345000 & 1.210448000 & -0.004204000 \\ 6 & 1.095256000 & -1.213826000 & -0.003812000 \\ 1 & 0.544546000 & 2.151802000 & -0.005769000 \\ 6 & 0.399111000 & -0.003085000 & -0.005816000 \\ 53 & -1.711824000 & -0.005475000 & -0.006922000 \\ 7 & -4.788627000 & 0.018058000 & 0.026380000 \\ 1 & -5.147898000 & -0.213431000 & 0.948691000 \\ 1 & -5.196776000 & -0.645477000 & -0.626692000 \\ 1 & -5.155839000 & 0.935002000 & -0.213767000\end{array}$


Supplementary Information

$\begin{array}{llll}1 & 3.039275000 & -2.149910000 & 0.002374000 \\ 1 & 0.553382000 & -2.157454000 & -0.005071000 \\ 6 & 4.621391000 & 0.005827000 & 0.008399000 \\ 7 & 5.778808000 & 0.008770000 & 0.013705000\end{array}$

\section{Ortho-Bisub-Iodobenzene-CN - - - $\mathrm{NH}_{3}$}

$\begin{array}{llll}6 & 3.675874000 & -0.025916000 & 0.005586000 \\ 6 & 2.986590000 & 1.181021000 & 0.003733000 \\ 6 & 2.966398000 & -1.221146000 & 0.003691000 \\ 1 & 3.522129000 & 2.128487000 & 0.005072000 \\ 6 & 1.585482000 & 1.194522000 & 0.000316000 \\ 6 & 1.565311000 & -1.210950000 & 0.000268000 \\ 6 & 0.849741000 & -0.002126000 & -0.001228000 \\ 53 & -1.257615000 & 0.012331000 & -0.002601000 \\ 7 & -4.221264000 & -0.002979000 & 0.010067000 \\ 1 & -4.596156000 & 0.243867000 & 0.922134000 \\ 1 & -4.581004000 & -0.925144000 & -0.221565000 \\ 1 & -4.616064000 & 0.650480000 & -0.661158000 \\ 1 & 4.763469000 & -0.035063000 & 0.008496000 \\ 1 & 3.485800000 & -2.177606000 & 0.005016000 \\ 6 & 0.881357000 & -2.480226000 & -0.001613000 \\ 7 & 0.372289000 & -3.519155000 & -0.003329000 \\ 6 & 0.922716000 & 2.474944000 & -0.001544000 \\ 7 & 0.431065000 & 3.522235000 & -0.003221000\end{array}$

\section{Meta-Bisub-Iodobenzene-CN - - - $\mathrm{NH}_{3}$}

$\begin{array}{llll}6 & 3.033447000 & 0.004897000 & 0.002817000\end{array}$

$\begin{array}{llll}6 & 2.316922000 & 1.203037000 & 0.000819000\end{array}$ 
Supplementary Information

$\begin{array}{lrrr}6 & 2.323217000 & -1.196975000 & 0.000968000 \\ 6 & 0.916415000 & 1.207082000 & -0.002858000 \\ 6 & 0.922671000 & -1.208598000 & -0.002725000 \\ 1 & 0.381649000 & 2.154629000 & -0.004003000 \\ 6 & 0.223925000 & -0.002569000 & -0.004522000 \\ 53 & -1.887719000 & -0.005705000 & -0.005733000 \\ 7 & -4.902053000 & 0.009248000 & 0.019294000 \\ 1 & -5.265704000 & -0.226962000 & 0.938846000 \\ 1 & -5.300036000 & -0.657568000 & -0.636935000 \\ 1 & -5.277994000 & 0.922458000 & -0.222221000 \\ 1 & 4.120461000 & 0.007741000 & 0.006035000 \\ 1 & 0.392999000 & -2.159021000 & -0.003687000 \\ 6 & 3.041568000 & -2.447466000 & 0.003537000 \\ 7 & 3.614978000 & -3.451996000 & 0.005865000 \\ 6 & 3.028266000 & 2.457520000 & 0.003422000 \\ 7 & 3.595527000 & 3.465540000 & 0.005849000\end{array}$




\section{Monomers}

\section{A. Unsubstituted Monomers}

\begin{tabular}{lrrr} 
& \multicolumn{3}{c}{ Alkene 1-H } \\
6 & 2.441869000 & -0.335773000 & -0.000005000 \\
6 & 1.442348000 & 0.537374000 & -0.000004000 \\
1 & 2.281042000 & -1.412231000 & 0.000012000 \\
1 & 1.579750000 & 1.616367000 & 0.000012000 \\
53 & -0.578029000 & -0.027232000 & 0.000000000 \\
1 & 3.469445000 & 0.029581000 & 0.000016000
\end{tabular}

Alkene 2-H

$6 \quad-1.687372000 \quad-0.149845000 \quad-0.000020000$

$\begin{array}{llll}6 & -0.617591000 & 0.650107000 & -0.000023000\end{array}$

$\begin{array}{llll}1 & -1.567669000 & -1.235197000 & -0.000014000\end{array}$

$\begin{array}{llll}1 & -0.683397000 & 1.736546000 & -0.000012000\end{array}$

$\begin{array}{llll}53 & 1.350194000 & -0.062139000 & 0.000002000\end{array}$

$\begin{array}{llll}6 & -3.051826000 & 0.376055000 & 0.000019000\end{array}$

$\begin{array}{llll}1 & -3.163772000 & 1.462572000 & 0.000018000\end{array}$

$\begin{array}{llll}6 & -4.136412000 & -0.407793000 & 0.000011000\end{array}$

$\begin{array}{llll}1 & -5.140137000 & 0.012604000 & -0.000013000\end{array}$

$1 \quad-4.046103000 \quad-1.494311000 \quad-0.000030000$
Alkene 3-H
$6 \quad 0.810507000$
$-0.327867000$
$-0.000944000$
$6 \quad-0.198695000$
0.531378000
0.179735000 
Supplementary Information

$\begin{array}{cccc}1 & 0.607964000 & -1.367205000 & -0.267797000 \\ 1 & -0.053250000 & 1.576799000 & 0.445142000 \\ 53 & -2.210640000 & -0.002010000 & -0.024967000 \\ 6 & 2.203911000 & 0.070993000 & 0.146116000 \\ 1 & 2.401201000 & 1.109283000 & 0.424600000 \\ 6 & 3.232706000 & -0.779952000 & -0.022668000 \\ 6 & 4.648437000 & -0.412991000 & 0.095868000 \\ 1 & 5.324218000 & -1.200772000 & 0.436320000 \\ 6 & 5.156794000 & 0.789796000 & -0.199252000 \\ 1 & 4.532990000 & 1.589253000 & -0.599390000 \\ 1 & 6.216595000 & 0.999002000 & -0.067591000 \\ 1 & 3.012233000 & -1.827971000 & -0.241185000\end{array}$

\section{Alkene 4-H}

$\begin{array}{lrrr}6 & -0.124519000 & 0.428858000 & -0.073318000 \\ 6 & -1.077738000 & -0.483513000 & 0.148847000 \\ 1 & -0.394285000 & 1.453634000 & -0.336244000 \\ 1 & -0.865153000 & -1.518217000 & 0.411162000 \\ 53 & -3.121854000 & -0.060730000 & 0.022070000 \\ 6 & 1.292277000 & 0.109989000 & 0.023586000 \\ 1 & 1.556205000 & -0.912348000 & 0.305206000 \\ 6 & 2.264588000 & 1.017490000 & -0.193862000 \\ 6 & 3.699349000 & 0.745523000 & -0.133958000 \\ 1 & 4.347105000 & 1.594260000 & 0.097234000 \\ 6 & 4.271694000 & -0.447113000 & -0.386859000 \\ 1 & 1.970976000 & 2.047271000 & -0.412696000 \\ 6 & 5.708430000 & -0.718627000 & -0.265979000 \\ 1 & 6.119970000 & -1.463186000 & -0.951200000\end{array}$


Supplementary Information

$\begin{array}{llll}6 & 6.523523000 & -0.138216000 & 0.623608000 \\ 1 & 7.587592000 & -0.365879000 & 0.644497000 \\ 1 & 6.144117000 & 0.565026000 & 1.365187000 \\ 1 & 3.646091000 & -1.268224000 & -0.745276000\end{array}$

Alkyne 1-H

$\begin{array}{llll}6 & 0.000410000 & -2.671874000 & 0.000000000\end{array}$

$\begin{array}{llll}6 & -0.000632000 & -1.462497000 & 0.000000000\end{array}$

$\begin{array}{llll}1 & 0.001331000 & -3.742372000 & 0.000000000\end{array}$

$\begin{array}{llll}53 & 0.000000000 & 0.538653000 & 0.000000000\end{array}$

Alkyne 2-H

$\begin{array}{llll}6 & 1.922391000 & 0.001274000 & 0.000115000 \\ 6 & 0.706993000 & 0.000857000 & -0.000495000 \\ 53 & -1.287736000 & -0.000119000 & 0.000032000 \\ 6 & 3.301563000 & -0.000866000 & -0.000099000 \\ 6 & 4.513512000 & 0.000319000 & 0.000240000 \\ 1 & 5.583255000 & -0.003209000 & -0.000278000\end{array}$

Alkyne 3-H

$\begin{array}{lrrr}6 & -1.028654000 & -0.003429000 & -0.000667000 \\ 6 & 0.188798000 & -0.001929000 & -0.000111000 \\ 53 & 2.180793000 & 0.000593000 & 0.000057000 \\ 6 & -2.399207000 & -0.018958000 & 0.000113000 \\ 6 & -3.617636000 & 0.022206000 & 0.000007000 \\ 6 & -4.990741000 & -0.000619000 & -0.000376000 \\ 6 & -6.203871000 & -0.001026000 & 0.000463000 \\ 1 & -7.274177000 & -0.008912000 & 0.000394000\end{array}$


Supplementary Information

\section{Iodobenzene}

$\begin{array}{lrrr}6 & -3.338108000 & -0.000036000 & 0.000027000 \\ 6 & -2.639103000 & 1.206125000 & 0.000004000 \\ 6 & -2.639026000 & -1.206181000 & 0.000004000 \\ 1 & -3.178726000 & 2.152285000 & 0.000034000 \\ 1 & -3.178611000 & -2.152361000 & 0.000034000 \\ 6 & -1.243953000 & 1.214456000 & -0.000028000 \\ 6 & -1.243892000 & -1.214418000 & -0.000028000 \\ 1 & -0.697288000 & 2.155350000 & -0.000005000 \\ 6 & -0.560595000 & 0.000064000 & -0.000047000 \\ 1 & -4.426812000 & -0.000056000 & 0.000038000 \\ 53 & 1.550314000 & 0.000000000 & 0.000006000 \\ 1 & -0.697155000 & -2.155267000 & -0.000005000\end{array}$

\section{B. Fluorinated Monomers}

\begin{tabular}{lrrr} 
& \multicolumn{3}{c}{ Alkene 1-F } \\
6 & -1.845717000 & -0.052563000 & 0.000076000 \\
6 & -0.756657000 & -0.803476000 & 0.000023000 \\
1 & -0.848334000 & -1.883797000 & -0.000039000 \\
53 & 1.152544000 & 0.009895000 & -0.000003000 \\
9 & -3.074390000 & -0.541485000 & -0.000034000 \\
9 & -1.883637000 & 1.263217000 & -0.000009000
\end{tabular}

Alkene 2-F

$\begin{array}{llll}6 & 0.907632000 & -0.070635000 & -0.000052000\end{array}$

$\begin{array}{llll}6 & -0.218731000 & -0.788761000 & -0.000004000\end{array}$

$\begin{array}{llll}1 & 0.867362000 & 1.018682000 & -0.000096000\end{array}$ 
Supplementary Information

$\begin{array}{lrrr}1 & -0.237422000 & -1.876883000 & 0.000031000 \\ 53 & -2.118923000 & 0.082717000 & 0.000008000 \\ 6 & 2.223706000 & -0.697205000 & -0.000060000 \\ 1 & 2.324544000 & -1.779701000 & -0.000019000 \\ 6 & 3.347208000 & 0.013170000 & -0.000062000 \\ 9 & 3.412467000 & 1.332607000 & 0.000011000 \\ 9 & 4.564149000 & -0.497666000 & 0.000073000\end{array}$

Alkene 3-F

$\begin{array}{lrrr}6 & -0.031677000 & 0.494398000 & 0.000129000 \\ 6 & -0.969035000 & -0.460573000 & 0.000488000 \\ 1 & -0.321925000 & 1.547175000 & -0.000390000 \\ 1 & -0.739999000 & -1.524569000 & 0.001039000 \\ 53 & -3.019606000 & -0.049323000 & -0.000080000 \\ 6 & 1.391517000 & 0.187109000 & 0.000397000 \\ 1 & 1.672850000 & -0.866172000 & 0.000883000 \\ 6 & 2.326997000 & 1.158310000 & -0.000002000 \\ 6 & 3.778348000 & 1.008324000 & 0.000080000 \\ 1 & 4.396516000 & 1.902245000 & 0.000162000 \\ 6 & 4.456164000 & -0.136269000 & -0.000053000 \\ 1 & 1.981801000 & 2.193702000 & -0.000449000 \\ 9 & 5.774422000 & -0.224728000 & -0.000094000 \\ 9 & 3.929580000 & -1.347054000 & -0.000267000\end{array}$

Alkene 4-F

$\begin{array}{llll}6 & 1.045202000 & 0.372414000 & 0.147621000\end{array}$

$\begin{array}{llll}6 & 2.010762000 & -0.481676000 & -0.212228000\end{array}$

$\begin{array}{llll}1 & 1.302727000 & 1.352759000 & 0.553966000\end{array}$ 
Supplementary Information

$\begin{array}{lrrr}1 & 1.813657000 & -1.470587000 & -0.622133000 \\ 53 & 4.048324000 & -0.042962000 & -0.044506000 \\ 6 & -0.367626000 & 0.049295000 & 0.017580000 \\ 1 & -0.618914000 & -0.926363000 & -0.406052000 \\ 6 & -1.349456000 & 0.904505000 & 0.366987000 \\ 6 & -2.782771000 & 0.632572000 & 0.281210000 \\ 1 & -3.432015000 & 1.500348000 & 0.167123000 \\ 6 & -3.320475000 & -0.601019000 & 0.367315000 \\ 1 & -1.064260000 & 1.898205000 & 0.721256000 \\ 6 & -4.728825000 & -0.968812000 & 0.256408000 \\ 1 & -5.020399000 & -1.992553000 & 0.476989000 \\ 6 & -5.729566000 & -0.172438000 & -0.109870000 \\ 1 & -2.651432000 & -1.440503000 & 0.564289000 \\ 9 & -5.625520000 & 1.102989000 & -0.436469000 \\ 9 & -6.991591000 & -0.553361000 & -0.206507000\end{array}$

Alkyne 1-F

$\begin{array}{lrrr}6 & -2.237849000 & -0.000317000 & 0.000136000 \\ 6 & -1.034238000 & -0.001314000 & -0.000062000 \\ 53 & 0.968034000 & 0.000093000 & -0.000001000 \\ 9 & -3.519254000 & 0.000542000 & -0.000046000\end{array}$

Alkyne 2-F

$\begin{array}{lrrr}6 & -1.382806000 & -0.002961000 & -0.000250000 \\ 6 & -0.167883000 & -0.004317000 & 0.001023000 \\ 53 & 1.828121000 & 0.000641000 & -0.000038000 \\ 6 & -2.762093000 & -0.001700000 & -0.000469000 \\ 6 & -3.967921000 & -0.001927000 & -0.000551000\end{array}$


Supplementary Information

$9 \quad-5.245132000 \quad 0.003496000 \quad 0.000387000$

Alkyne 3-F

$\begin{array}{lrrr}6 & -0.416982000 & -0.012835000 & 0.001932000 \\ 6 & 0.800317000 & -0.007975000 & 0.004756000 \\ 53 & 2.792752000 & 0.002667000 & -0.000144000 \\ 6 & -1.788245000 & -0.028626000 & -0.003187000 \\ 6 & -3.005881000 & 0.014804000 & -0.002821000 \\ 6 & -4.379189000 & -0.002170000 & -0.003282000 \\ 6 & -5.586004000 & 0.007766000 & -0.001116000 \\ 9 & -6.862213000 & 0.003651000 & 0.003326000\end{array}$

Ortho-Iodobenzene-F

$\begin{array}{llll}6 & -3.283898000 & -0.307900000 & -0.000009000\end{array}$

$\begin{array}{llll}6 & -2.545701000 & -1.491388000 & -0.000008000\end{array}$

$\begin{array}{llll}6 & -2.632850000 & 0.922928000 & 0.000001000\end{array}$

$\begin{array}{llll}1 & -3.052812000 & -2.454473000 & -0.000050000\end{array}$

$\begin{array}{llll}1 & -3.176307000 & 1.865780000 & -0.000001000\end{array}$

$\begin{array}{llll}6 & -1.152651000 & -1.448993000 & 0.000020000\end{array}$

$\begin{array}{llll}6 & -1.244709000 & 0.949170000 & -0.000009000\end{array}$

$\begin{array}{llll}1 & -0.572399000 & -2.369697000 & -0.000016000\end{array}$

$\begin{array}{llll}6 & -0.491996000 & -0.220408000 & 0.000041000\end{array}$

$\begin{array}{llll}1 & -4.371869000 & -0.340181000 & 0.000002000\end{array}$

$\begin{array}{llll}53 & 1.601968000 & -0.120496000 & -0.000003000\end{array}$

$\begin{array}{llll}9 & -0.624455000 & 2.140487000 & 0.000001000\end{array}$

Meta-Iodobenzene-F

$\begin{array}{llll}6 & -3.012117000 & 0.708004000 & 0.000029000\end{array}$ 
Supplementary Information

$\begin{array}{lrrr}6 & -2.111767000 & 1.771443000 & 0.000008000 \\ 6 & -2.497661000 & -0.580014000 & -0.000006000 \\ 1 & -2.486306000 & 2.793867000 & 0.000054000 \\ 6 & -0.734576000 & 1.547583000 & -0.000037000 \\ 6 & -1.135583000 & -0.850116000 & -0.000065000 \\ 1 & -0.040047000 & 2.383914000 & 0.000026000 \\ 6 & -0.263293000 & 0.235925000 & -0.000068000 \\ 53 & 1.813152000 & -0.123754000 & 0.000010000 \\ 1 & -4.088867000 & 0.860375000 & 0.000046000 \\ 1 & -0.785737000 & -1.879304000 & -0.000039000 \\ 9 & -3.351790000 & -1.621874000 & 0.000026000\end{array}$

Para-Iodobenzene-F

$\begin{array}{llll}6 & 2.939878000 & -0.000018000 & 0.000012000\end{array}$

$\begin{array}{llll}6 & 2.272889000 & -1.215972000 & 0.000010000\end{array}$

$\begin{array}{llll}6 & 2.272952000 & 1.215953000 & 0.000010000\end{array}$

$\begin{array}{llll}1 & 2.840162000 & -2.144371000 & 0.000039000\end{array}$

$\begin{array}{llll}1 & 2.840265000 & 2.144328000 & 0.000039000\end{array}$

$\begin{array}{llll}6 & 0.878659000 & -1.213317000 & -0.000019000\end{array}$

$\begin{array}{llll}6 & 0.878712000 & 1.213384000 & -0.000019000\end{array}$

$\begin{array}{llll}1 & 0.334309000 & -2.155351000 & 0.000016000\end{array}$

$\begin{array}{llll}6 & 0.192301000 & 0.000068000 & -0.000046000\end{array}$

$9 \quad 4.287816000 \quad-0.000042000 \quad-0.000001000$

$\begin{array}{llll}53 & -1.916072000 & -0.000005000 & 0.000004000\end{array}$

$\begin{array}{llll}1 & 0.334420000 & 2.155455000 & 0.000016000\end{array}$

Ortho-Bisub-Iodobenzene-F

$\begin{array}{llll}6 & 3.252534000 & 0.000012000 & -0.000052000\end{array}$ 
Supplementary Information

$\begin{array}{llll}6 & 2.563978000 & 1.210669000 & -0.000020000 \\ 6 & 2.563990000 & -1.210655000 & -0.000020000 \\ 1 & 3.077221000 & 2.169514000 & -0.000041000 \\ 1 & 3.077245000 & -2.169492000 & -0.000040000 \\ 6 & 1.176394000 & 1.188072000 & 0.000030000 \\ 6 & 1.176408000 & -1.188074000 & 0.000032000 \\ 6 & 0.449377000 & -0.000004000 & 0.000039000 \\ 1 & 4.340598000 & 0.000023000 & -0.000094000 \\ 53 & -1.638129000 & -0.000002000 & -0.000015000 \\ 9 & 0.512773000 & -2.352931000 & 0.000050000 \\ 9 & 0.512749000 & 2.352926000 & 0.000050000\end{array}$

Meta-Bisub-Iodobenzene-F

$\begin{array}{llll}6 & 2.871904000 & 0.000039000 & 0.000046000\end{array}$

$\begin{array}{llll}6 & 2.141694000 & -1.179540000 & 0.000008000\end{array}$

$\begin{array}{llll}6 & 2.141632000 & 1.179588000 & 0.000009000\end{array}$

$\begin{array}{lllll}6 & 0.753313000 & -1.217987000 & -0.000052000\end{array}$

$\begin{array}{llll}6 & 0.753249000 & 1.217928000 & -0.000051000\end{array}$

$\begin{array}{llll}1 & 0.235713000 & -2.173518000 & -0.000012000\end{array}$

$\begin{array}{llll}6 & 0.076713000 & -0.000058000 & -0.000052000\end{array}$

$\begin{array}{llll}53 & -2.027995000 & -0.000009000 & 0.000009000\end{array}$

$\begin{array}{llll}1 & 3.958289000 & 0.000027000 & 0.000055000\end{array}$

$\begin{array}{llll}1 & 0.235583000 & 2.173424000 & -0.000012000\end{array}$

$\begin{array}{llll}9 & 2.812326000 & 2.344421000 & 0.000003000\end{array}$

$\begin{array}{llll}9 & 2.812468000 & -2.344339000 & 0.000004000\end{array}$

\section{C. Cyanated Monomers}


Supplementary Information

\begin{tabular}{cccc}
\multicolumn{4}{c}{ Alkene 1-CN } \\
6 & 1.578254000 & -0.059376000 & -0.000184000 \\
6 & 0.479454000 & -0.837767000 & -0.000059000 \\
1 & 0.579422000 & -1.921682000 & -0.000086000 \\
53 & -1.455788000 & -0.120817000 & 0.000187000 \\
6 & 1.524712000 & 1.378405000 & -0.000191000 \\
6 & 2.882616000 & -0.672477000 & -0.000372000 \\
7 & 1.476602000 & 2.533575000 & -0.000186000 \\
7 & 3.921559000 & -1.180389000 & -0.000527000
\end{tabular}

Alkene 2-CN

$\begin{array}{lrrr}6 & 0.528401000 & -0.068464000 & 0.000036000 \\ 6 & -0.592411000 & -0.805614000 & -0.000092000 \\ 1 & 0.490319000 & 1.021562000 & 0.000165000 \\ 1 & -0.578742000 & -1.895056000 & -0.000209000 \\ 53 & -2.499807000 & 0.014890000 & 0.000004000 \\ 6 & 1.821477000 & -0.718171000 & 0.000039000 \\ 1 & 1.850890000 & -1.808589000 & 0.000018000 \\ 6 & 3.005937000 & -0.057700000 & 0.000048000 \\ 6 & 3.065623000 & 1.379514000 & 0.000020000 \\ 6 & 4.250857000 & -0.775641000 & -0.000003000 \\ 7 & 3.077336000 & 2.536351000 & -0.000060000 \\ 7 & 5.243809000 & -1.369299000 & -0.000011000\end{array}$

Alkene 3-CN

$\begin{array}{llll}6 & 0.442966000 & -0.614780000 & 0.000029000\end{array}$

$\begin{array}{llll}6 & 1.350138000 & 0.372901000 & 0.003015000\end{array}$

$1 \quad 0.758643000 \quad-1.659270000 \quad-0.003807000$ 
Supplementary Information

$\begin{array}{lrrr}1 & 1.076705000 & 1.427219000 & 0.006935000 \\ 53 & 3.404190000 & 0.030468000 & -0.000181000 \\ 6 & -0.978191000 & -0.325581000 & 0.001659000 \\ 1 & -1.264476000 & 0.725958000 & 0.005587000 \\ 6 & -1.908137000 & -1.311927000 & -0.001601000 \\ 6 & -3.348407000 & -1.174649000 & -0.000481000 \\ 1 & -3.905667000 & -2.112045000 & 0.000005000 \\ 6 & -4.123692000 & -0.056338000 & -0.000366000 \\ 1 & -1.552830000 & -2.343053000 & -0.005055000 \\ 6 & -3.606412000 & 1.281735000 & -0.001453000 \\ 6 & -5.556018000 & -0.182332000 & 0.000777000 \\ 7 & -3.173532000 & 2.355546000 & -0.002173000 \\ 7 & -6.707601000 & -0.296659000 & 0.001668000\end{array}$

\section{Alkene 4-CN}

$\begin{array}{lrrr}6 & 1.503522000 & 0.363475000 & 0.136422000 \\ 6 & 2.468660000 & -0.510351000 & -0.179751000 \\ 1 & 1.756378000 & 1.365971000 & 0.485356000 \\ 1 & 2.262111000 & -1.519478000 & -0.532427000 \\ 53 & 4.502323000 & -0.072855000 & -0.040834000 \\ 6 & 0.097673000 & 0.016245000 & 0.031756000 \\ 1 & -0.136713000 & -0.988124000 & -0.328767000 \\ 6 & -0.897558000 & 0.879803000 & 0.329015000 \\ 6 & -2.319934000 & 0.586831000 & 0.243513000 \\ 1 & -2.975013000 & 1.455471000 & 0.198754000 \\ 6 & -2.851738000 & -0.661056000 & 0.246956000 \\ 1 & -0.631698000 & 1.895935000 & 0.624775000 \\ 6 & -4.248278000 & -1.021468000 & 0.148713000\end{array}$


Supplementary Information

$\begin{array}{llll}1 & -4.458594000 & -2.088459000 & 0.228561000 \\ 6 & -5.351417000 & -0.243110000 & -0.029769000 \\ 1 & -2.180202000 & -1.511814000 & 0.362437000 \\ 6 & -5.320985000 & 1.184310000 & -0.168972000 \\ 6 & -6.650689000 & -0.856129000 & -0.088630000 \\ 7 & -5.285817000 & 2.336565000 & -0.277781000 \\ 7 & -7.690603000 & -1.362205000 & -0.135076000\end{array}$

Alkyne 1-CN

$\begin{array}{lrrr}6 & -1.936320000 & 0.000925000 & 0.000073000 \\ 6 & -0.722809000 & 0.000600000 & -0.000148000 \\ 53 & 1.268424000 & -0.000096000 & 0.000006000 \\ 6 & -3.319759000 & 0.000250000 & 0.000041000 \\ 7 & -4.479019000 & -0.000791000 & -0.000019000\end{array}$

Alkyne 2-CN

$\begin{array}{llll}6 & 1.043004000 & 0.001317000 & -0.000348000 \\ 6 & -0.174075000 & 0.000054000 & -0.002795000 \\ 53 & -2.164113000 & -0.000590000 & 0.000234000 \\ 6 & 2.413228000 & 0.019218000 & 0.000327000 \\ 6 & 3.629063000 & -0.011929000 & 0.000078000 \\ 6 & 5.008520000 & -0.004808000 & 0.000721000 \\ 7 & 6.168507000 & 0.001162000 & -0.000045000\end{array}$

Alkyne 3-CN

$\begin{array}{llll}6 & -0.055410000 & -0.018338000 & -0.012140000\end{array}$

$6 \quad \begin{array}{llll}6 & 1.162531000 & -0.007562000 & -0.006658000\end{array}$

$\begin{array}{llll}53 & 3.152182000 & 0.003640000 & 0.001299000\end{array}$ 
Supplementary Information

$\begin{array}{llll}6 & -1.422631000 & -0.002729000 & -0.019188000 \\ 6 & -2.642204000 & -0.012205000 & 0.025434000 \\ 6 & -4.006472000 & -0.026545000 & 0.001359000 \\ 6 & -5.223082000 & 0.009943000 & 0.004918000 \\ 6 & -6.601616000 & 0.010401000 & 0.001390000 \\ 7 & -7.761761000 & 0.012753000 & -0.005645000\end{array}$

Ortho-Iodobenzene-CN

$\begin{array}{llll}6 & 3.290984000 & -0.524806000 & 0.000007000\end{array}$

$\begin{array}{llll}6 & 2.517817000 & -1.685701000 & -0.000006000\end{array}$

$\begin{array}{llll}6 & 2.669139000 & 0.717433000 & 0.000009000\end{array}$

$\begin{array}{llll}1 & 2.998573000 & -2.663048000 & -0.000008000\end{array}$

$6 \quad 1.125936000 \quad-1.610115000 \quad-0.000011000$

$\begin{array}{llll}6 & 1.269761000 & 0.806095000 & -0.000003000\end{array}$

$\begin{array}{llll}1 & 0.526755000 & -2.517875000 & -0.000022000\end{array}$

$\begin{array}{llll}6 & 0.498390000 & -0.366079000 & -0.000010000\end{array}$

$\begin{array}{llll}53 & -1.600564000 & -0.279183000 & 0.000002000\end{array}$

$1 \quad 4.377284000 \quad-0.587148000 \quad 0.000018000$

$\begin{array}{llll}1 & 3.253385000 & 1.635548000 & 0.000022000\end{array}$

$\begin{array}{llll}6 & 0.663807000 & 2.112476000 & 0.000013000\end{array}$

$\begin{array}{llll}7 & 0.208408000 & 3.176201000 & -0.000019000\end{array}$

Meta-Iodobenzene-CN

$\begin{array}{llll}6 & 2.668098000 & 1.248526000 & -0.000001000\end{array}$

$\begin{array}{llll}6 & 1.625288000 & 2.169059000 & 0.000000000\end{array}$

$\begin{array}{llll}6 & 2.371894000 & -0.118727000 & 0.000000000\end{array}$

$\begin{array}{llll}1 & 1.842780000 & 3.235733000 & 0.000001000\end{array}$

$\begin{array}{llll}6 & 0.298321000 & 1.738486000 & 0.000001000\end{array}$ 
Supplementary Information

$\begin{array}{llll}6 & 1.045974000 & -0.569265000 & 0.000001000 \\ 1 & -0.513922000 & 2.462494000 & 0.000005000 \\ 6 & 0.021166000 & 0.371181000 & 0.000000000 \\ 53 & -1.978576000 & -0.286736000 & 0.000000000 \\ 1 & 3.705253000 & 1.576432000 & -0.000002000 \\ 1 & 0.834301000 & -1.635959000 & 0.000005000 \\ 6 & 3.444049000 & -1.083094000 & 0.000000000 \\ 7 & 4.306770000 & -1.854100000 & 0.000000000\end{array}$

Para-Iodobenzene-CN

$\begin{array}{llll}6 & 2.709229000 & 0.000024000 & 0.000050000 \\ 6 & 2.013721000 & -1.214067000 & 0.000048000 \\ 6 & 2.013766000 & 1.214112000 & 0.000048000 \\ 1 & 2.563965000 & -2.152851000 & 0.000093000 \\ 6 & 0.622454000 & -1.215295000 & 0.000032000 \\ 6 & 0.622492000 & 1.215395000 & 0.000032000 \\ 1 & 0.077156000 & -2.156470000 & 0.000099000 \\ 6 & -0.062281000 & 0.000083000 & 0.000011000 \\ 53 & -2.167017000 & -0.000016000 & -0.000016000 \\ 1 & 2.564030000 & 2.152886000 & 0.000093000 \\ 1 & 0.077231000 & 2.156595000 & 0.000098000 \\ 6 & 4.150239000 & -0.000018000 & -0.000028000 \\ 7 & 5.307398000 & -0.000100000 & -0.000102000\end{array}$

Ortho-Bisub-Iodobenzene-CN

$\begin{array}{llll}6 & -3.297890000 & -0.000162000 & -0.000014000\end{array}$

$\begin{array}{llll}6 & -2.599744000 & -1.201855000 & -0.000007000\end{array}$

$\begin{array}{llll}6 & -2.599829000 & 1.201591000 & -0.000007000\end{array}$ 
Supplementary Information

$\begin{array}{lrrr}1 & -3.127163000 & -2.153781000 & -0.000011000 \\ 6 & -1.198627000 & -1.206966000 & -0.000004000 \\ 6 & -1.198714000 & 1.206816000 & -0.000004000 \\ 6 & -0.482247000 & -0.000052000 & -0.000003000 \\ 53 & 1.611351000 & 0.000081000 & -0.000008000 \\ 1 & -4.385478000 & -0.000202000 & -0.000018000 \\ 1 & -3.127334000 & 2.153467000 & -0.000010000 \\ 6 & -0.522768000 & 2.478920000 & 0.000002000 \\ 7 & -0.016507000 & 3.518988000 & 0.000047000 \\ 6 & -0.522493000 & -2.478975000 & 0.000002000 \\ 7 & -0.016027000 & -3.518942000 & 0.000047000\end{array}$

Meta-Bisub-Iodobenzene-CN

$\begin{array}{lrrr}6 & -2.597473000 & 0.000015000 & -0.000003000 \\ 6 & -1.885741000 & 1.201020000 & 0.000001000 \\ 6 & -1.885775000 & -1.200998000 & 0.000001000 \\ 6 & -0.485357000 & 1.211589000 & 0.000008000 \\ 6 & -0.485380000 & -1.211603000 & 0.000009000 \\ 1 & 0.048918000 & 2.158934000 & -0.000010000 \\ 6 & 0.200663000 & -0.000022000 & 0.000019000 \\ 53 & 2.303187000 & -0.000010000 & -0.000002000 \\ 1 & -3.684571000 & 0.000025000 & -0.000011000 \\ 1 & 0.048863000 & -2.158967000 & -0.000008000 \\ 6 & -2.601300000 & -2.452943000 & -0.000002000 \\ 7 & -3.173793000 & -3.457750000 & -0.000004000 \\ 6 & -2.601230000 & 2.452983000 & -0.000002000 \\ 7 & -3.173717000 & 3.457794000 & -0.000003000\end{array}$


Supplementary Information

\section{D. Ammonia Monomer}

\begin{tabular}{crrr}
\multicolumn{3}{c}{ Ammonia } \\
7 & 0.000000000 & 0.000000000 & 0.114945000 \\
1 & 0.000000000 & 0.941721000 & -0.268204000 \\
1 & -0.815555000 & -0.470861000 & -0.268204000 \\
1 & 0.815555000 & -0.470861000 & -0.268204000
\end{tabular}

